\title{
Classifying by colors: FISH-based genome analysis
}

\author{
C. Fauth and M.R. Speicher
}

In Fig. $4 \mathrm{~b}$ the chromosomes in the lower row, "visual" column were depicted incorrectly. Due to the wrong illustration it cannot be understood why the shown translocation should be identified with both high specificity and sensitivity. The correct Fig. 4 with its corresponding legend is shown here.

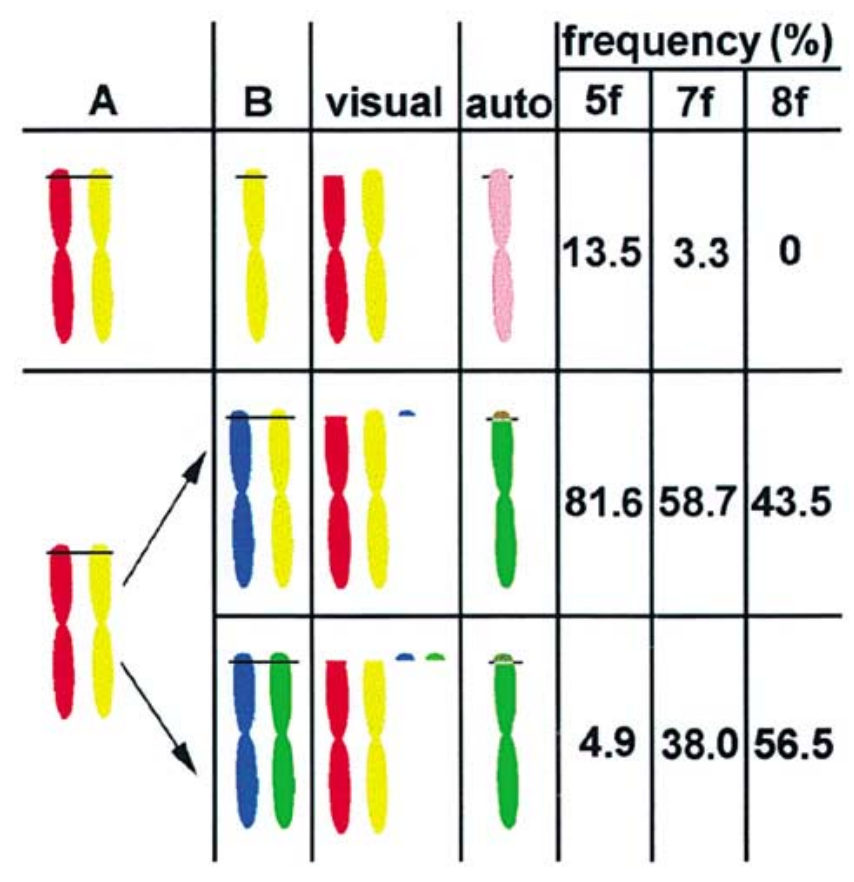

Fig. 4. Scheme illustrating the impact of probe design on the accurate identification and classification of small interchromosomal rearrangements. It is shown exemplarily for translocations, but the same considerations apply to small insertions as well. There are 552 possible two-way translocations in a male metaphase spread. If small ( $\sim 3 \mathrm{Mb}$ or less), the design of the probe mix determines critically the number of translocations, which can be detected and correctly classified. (a) Illustration of a small translocation, which has both a low sensitivity and a low specificity. Chromosome A is labeled with two fluorochromes (red and yellow), chromosome B is labeled with the yellow fluorochrome only. If part of chromosome B is translocated to chromosome A, the translocated segment is only labeled with a fluorochrome that also occurs in the centric segment. A small translocation may be completely obscured by fluorescence flaring and hence escape both, the visual inspection of individual fluorochrome channels (visual) and the automated classification (auto). Thus, a small interchromosomal rearrangement may be overlooked simply because of the fluorochrome composition in rearranged chromosomes. With the commonly used 24 color-painting kits, which employ five different fluorochromes, $13.5 \%$ out of all possible 552 translocations may be overlooked for this reason (5f). Using an optimized seven fluorochrome probe set, only $3.3 \%$ of all possible small interchromosomal rearrangements are overlooked because of the labeling (7f). If all chromosomes are labeled each with two different fluorochromes by using eight fluorochromes, this situation is completely avoided (8f) (for details see Azofeifa et al., 2000 and text). (b) A small translocation is in general much easier to identify if the translocated segment adds an extra fluorochrome to the centric segment. Two examples are depicted. In the first example shown in the upper row, the two chromosomes involved in the translocation have one fluorochrome in common. The translocation will be identified with high sensitivity but low specificity because fluorescence flaring may hamper distinguishing between the possible double combinations blue/yellow or blue/red. The frequencies for these high sensitivity/low specificity translocations are $81.6 \%$ (five fluorochrome mix), $58.7 \%$ (seven fluorochrome mix), and $43.5 \%$ (eight fluorochrome mix), respectively. The lower row shows an example in which both the translocated and the centric segment are labeled each with two different fluorochromes. If the analysis is done with the eight fluorochrome mix the translocation will be identified in any case unequivocally and cannot be obscured by fluorescence flaring. Translocations, which can be identified with a high specificity and sensitivity due to unique fluorochrome combinations occur with frequencies of $4.9 \%(5 \mathrm{f}), 38.0 \%(7 \mathrm{f})$, and $56.5 \%(8 \mathrm{f})$, respectively. 